\title{
BOREL MEASURABLE MAPPINGS FOR NONSEPARABLE METRIC SPACES
}

\author{
BY \\ R. W. HANSELL( $\left.{ }^{1}\right)$
}

\begin{abstract}
The main object of this paper is the extension of part of the basic theory of Borel measurable mappings, from the "classical" separable metric case, to general metric spaces. Although certain results of the standard theory are known to fail in the absence of separability, we show that they continue to hold for the class of " $\sigma$ discrete" mappings. This class is shown to be quite extensive, containing the continuous mappings, all mappings with a separable range, and any Borel measurable mappings whose domain is a Borel subset of a complete metric space. The last result is a consequence of our Basic Theorem which gives a topological characterization of those collections which are the inverse image of an open discrete collection under a Borel measurable mapping. Such collections are shown to possess a strong type of $\sigma$-discrete refinement.

The properties of $\sigma$-discrete mappings together with the known properties of "locally Borel" sets allow us to extend, to general metric spaces, well-known techniques used for separable spaces. The basic properties of "complex" and "product" mappings, well known for separable spaces, are proved for general metric spaces for the class of $\sigma$-discrete mappings. A consequence of these is a strengthening of the basic theorem of the structure theory of nonseparable Borel sets due to A. H. Stone. Finally, the classical continuity properties of Borel measurable mappings are extended, and, in particular, a generalization of the famous theorem of Baire on the points of discontinuity of a mapping of class 1 is obtained.
\end{abstract}

Introduction. In this paper we are primarily concerned with extending part of the "classical" (separable metric) theory of Borel measurable mappings to general metric spaces. Some work of this nature has already been done-notably the results of Montgomery in [10], the related work of Kuratowski in [7], and the work of Stone in [13]. It has long been suspected (and in some cases known, e.g. [13, p. 29] and $[6$, p. 488]) that certain basic results of the standard theory will not extend to nonseparable spaces, at least without imposing some further conditions upon the mappings (or perhaps the spaces themselves). Here we consider mappings $f: X \rightarrow Y$ which are " $\sigma$-discrete" in the sense that there exists a $\sigma$-discrete collection $\mathscr{B}$ of subsets of $X$ such that to each open $V \subset Y$ there corresponds a subcollection $\mathscr{B}_{V}$ of

Received by the editors June 17, 1970.

AMS 1969 subject classifications. Primary 0440, 2820, 5460; Secondary 2810, 5435.

Key words and phrases. Borel measurable mappings, descriptive set theory, Borel classifications, analytic sets, $\sigma$-discrete collections, $\sigma$-discrete sets, $\sigma$-discrete mappings, metric spaces.

(1) The results presented in this paper form a part of the author's doctoral dissertation at the University of Rochester, written under the direction of Professor A. H. Stone. 
$\mathscr{B}$ such that $f^{-1}(V)=\bigcup \mathscr{B}_{V}$. We call $\mathscr{B}$ a $\sigma$-discrete base for $f$. Note that all continuous mappings (with a metrizable range or domain) and all mappings with a separable range are $\sigma$-discrete. (See $\$ 3.2$ for further examples, and characterizations of those spaces $X$ and $Y$ for which every mapping $f: X \rightarrow Y$ is $\sigma$-discrete.)

The properties of $\sigma$-discrete mappings allow us to extend, to general metric spaces, well-known methods used for separable spaces. Thus we show that, to a large extent, $\sigma$-discrete bases (for mappings) can replace countable bases in the classical arguments. This in turn is largely a consequence of the properties of "locally Borel" sets (see e.g. [10] and $\$ 0.4$ of this paper). Our principal result regarding $\sigma$-discrete mappings, indicating their general nature, states that every Borel measurable (in fact, every analytic) mapping defined on an absolutely analytic domain is $\sigma$-discrete (Theorem 3, §3.2).

In more detail, the paper proceeds as follows. $\$ 0$ deals mainly with preliminaries and background material. In $\S 1$ we introduce and study the properties of a " $\sigma$ discretely decomposable" collection of sets-a collection $\left\{A_{d} \mid d \in D\right\}$ of subsets of a fixed space $X$ is said to be $\sigma$-discretely decomposable (abbrev. $\sigma$-d.d.) provided each $A_{d}=\bigcup A_{d n}(n=1,2, \ldots)$ such that, for fixed $n,\left\{A_{d n} \mid d \in D\right\}$ is discrete in $X$. In particular, we show that the definition is independent of the containing space $X$ (§1.3). For separable spaces, a disjoint collection is $\sigma$-d.d. iff it is countable. In $\$ 2$ we are mainly concerned with the study of disjoint collections of analytic sets which are "completely additive-absolutely analytic" in the sense that the union of every subcollection is absolutely analytic. Such collections arise naturally in the study of Borel measurable mappings (see $\$ 2.1$ ). Fundamental to what follows, we prove here our Basic Theorem (Theorem 2, \$2.7): A disjoint collection of analytic sets in a complete metric space $X$ is completely additive-analytic in $X$ if and only if it is $\sigma$-d.d. This theorem is then used in $\S 3$ to prove (Theorem $3, \S 3.2$ ) that every analytic mapping (respectively, Borel measurable mapping of class $\alpha\left(<\omega_{1}\right)$ ) defined on an absolutely analytic space is $\sigma$-discrete and has a $\sigma$-discrete base of analytic sets (resp. Borel measurable sets of class $\alpha$ ). The basic properties of "complex" and "product" mappings (\$3.5), well known for separable spaces, are then proven for general metric spaces for the class of $\sigma$-discrete mappings. As an application of these results, we obtain a sharpening (Theorem 8) of the "basic theorem" for the structure theory of nonseparable Borel sets due to A. H. Stone (see §3.6). The paper concludes (§3.7) with a generalization (Theorem 10) to nonseparable spaces of a famous theorem of $\mathbf{R}$. Baire on the points of discontinuity of a Borel measurable mapping of the first class [4, p. 289].

0. Preliminaries. For the convenience of the reader we collect here some of the more basic definitions, properties, and notational practices to be used in the sequel. Our terminology regarding Borel sets follows that of Kuratowski [6]; for analytic sets we follow Sierpiński [12]. The basic reference for general topology and axiomatic set theory is Kelley [5]. Throughout this paper, all spaces considered 
are assumed to be metrisable. We use the axiom of choice without specific mention, but not the continuum hypothesis.

0.1 Analytic sets. Let $X$ be a space and suppose that to each finite sequence $\left(n_{1}, \ldots, n_{p}\right)$ of natural numbers there corresponds a closed subset $F_{n_{1} \ldots n_{p}}$ of $X$. Then the set

$$
A=\bigcup_{\left(n_{p}\right)} \bigcap_{p=1}^{\infty} F_{n_{1} \ldots n_{p}}
$$

where the union is over all sequences $\left(n_{p}\right)=\left(n_{1}, n_{2}, \ldots\right)$ of natural numbers, is called an analytic (=Suslin [4, p. 203], $=\aleph_{0}$-analytic [13, p. 34]) set in $X$.

Analytic sets can be "regularized" in the sense of the following lemma (this result is essentially known, e.g. cf. [4, p. 205]).

LEMMA 1. To each analytic set $A$ in $X$ we can associate a family $\left\{A_{n_{1} \ldots n_{p}}\right\}$ of analytic sets in $X$ indexed by the finite sequences of natural numbers such that

(1) $A=A_{1} \cup A_{2} \cup \cdots$,

(2) $A_{n_{1} \ldots n_{p}}=A_{n_{1} \ldots n_{p} 1} \cup A_{n_{1} \ldots n_{p} 2} \cup \cdots$,

(3) $A=\bigcup_{\left(n_{p}\right)} \bigcap_{p=1}^{\infty} \bar{A}_{n_{1} \ldots n_{p}}($ closure in $X)$.

Proof. By hypothesis, we have

$$
A=\bigcup_{\left(n_{q}^{\prime}\right)} \bigcap_{q=1}^{\infty} F_{n_{1}^{\prime} \ldots n_{q}^{\prime}}
$$

for suitable closed subsets $F_{n_{1}^{\prime} \ldots n_{q}^{\prime}}$ of $X$. We now define $A_{n_{1} \ldots n_{p}}$ to be the union of all the sets $\bigcap_{q=1}^{\infty} F_{n_{1}^{\prime} \ldots n_{q}^{\prime}}$ with $n_{r}^{\prime}=n_{r}$ for $r=1, \ldots, p$. Then properties (1) and (2) are easily seen to hold (cf. [4, p. 205]). To prove (3), we note first that (1) and (2) together imply that $A \subset \bigcup_{\left(n_{p}\right)} \bigcap_{p=1}^{\infty} A_{n_{1} \ldots n_{p}}$, and hence, all the more so, is $A$ contained in the right side of (3). On the other hand, if $\left(n_{1}, \ldots, n_{p}, \ldots\right)$ is a given sequence of natural numbers, then, for each $p$, we have

$$
\bar{A}_{n_{1} \ldots n_{p}} \subset F_{n_{1} \ldots n_{p}}
$$

as follows easily from the definition of the sets $A_{n_{1} \ldots n_{p}}$ and the fact that the sets $F_{n_{1} \ldots n_{p}}$ are closed in $X$. Consequently, $\bigcap \bar{A}_{n_{1} \ldots n_{p}}(p=1,2, \ldots)$ is contained in $\bigcap F_{n_{1} \ldots n_{p}}(p=1,2, \ldots)$, from which it follows that the right side of (3) is contained in $A$. Thus equality in (3) must hold, and this completes the proof of the lemma.

0.2 Borel sets. The open (closed) subsets of a space $X$ are said to be of additive (multiplicative) class 0 in $X$. If $\alpha$ is a countable ordinal $>0$, a subset of $X$ is said to be of additive (multiplicative) class $\alpha$ in $X$ if it is the union (intersection) of a countable family of subsets of classes $<\alpha$ in $X$. The family of all sets of classes $<\omega_{1}$ (=first uncountable ordinal) in $X$ is the family of Borel subsets of $X$. Further, a subset of $X$ which is of both additive and multiplicative class $\alpha$ is said to be of ambiguous class $\alpha$ (in $X$ ). (Cf. [6, pp. 344-348].)

0.3 Sets with the Baire property. A subset $B$ of a metric space $X$ is said to have 
the Baire property in $X$ if $B=(G \sim P) \cup R$ where $G$ is open and $P, R$ are of the first category in $X$ (cf. [6, pp. 87-92]).

0.4 General properties of analytic, Borel, and Baire property sets. The following properties are well known.

(0) For a fixed metric space $X$, Borel in $X$ implies analytic in $X$ implies the Baire property in $X$. (See e.g. [12, p. 213] and [6, p. 482].)

(1) If $E \subset Y \subset X, E$ is analytic (Borel of additive [multiplicative] class $\alpha$ ) in $Y$ if and only if it is of the form $Y \cap M$ where $M$ has the corresponding property in $X$. (Cf. [13, p. 9].)

(2) If $E \subset X$, and $E$ is homeomorphic to a set which is analytic (Borel of additive class $\alpha>1$ [multiplicative class $\alpha \geqq 1$ ]) in some complete metric space, then $E$ has the same property in $X$. (See [6, p. 432] and [13, p. 36].)

Sets $E$ with this property are said to be absolutely analytic (absolutely Borel, etc.). From (1) above we see that an analytic (Borel) subset of an absolutely analytic (absolutely Borel) set is itself absolutely analytic (absolutely Borel).

(3) Countable unions (resp. intersections) of analytic (analytic) sets, additive (multiplicative) class $\alpha$ sets, and sets having the Baire property (Baire property) have the same property. (Cf. [13, p. 34] and [6, pp. 88, 344].)

(4) If $E \subset X$ is locally analytic( ${ }^{2}$ ) (Borel of additive class $\alpha$ [multiplicative class $\alpha>0$ ] has the Baire property) in $X$, then $E$ has the corresponding (global) property in $X$. (See [13, p. 35], [10], and [6, p. 361].)

(5) The union of a discrete (see $\$ 1.1$ for the definition) collection of analytic sets (Borel sets of some fixed additive or multiplicative class $\geqq 0$, sets having the Baire property) in $X$ has itself this property.

This follows immediately from (4) and a well-known [5, p. 126] property of discrete families of closed sets.

(6) A separable metric space has at most $2^{\aleph_{0}}$ analytic subsets. (See e.g. [13, Theorem $\left.23\left(k=\boldsymbol{\aleph}_{0}\right)\right]$.)

(7) If $B \subset X$ is a set of ambiguous class $\alpha>1$, then there exists a sequence of sets $B_{n}$ of ambiguous classes $<\alpha$ in $X$ such that

$$
B=\bigcup_{n=1}^{\infty}\left(B_{n} \cap B_{n+1} \cap \cdots\right)=\bigcap_{n=1}^{\infty}\left(B_{n} \cup B_{n+1} \cup \cdots\right) .
$$

In addition, if $\alpha=\lambda+1$, where $\lambda$ is a limit ordinal, the $B_{n}$ 's can be taken to be of class $<\lambda$. (See $[6$, pp. 355, 357].)

0.5 Analytic and Borel measurable mappings, and mappings with the Baire property. A mapping $f: X \rightarrow Y$ is analytic (resp. Borel measurable of class $\alpha$, has the Baire property) if for each open set $V \subset Y, f^{-1}(V)$ is analytic (resp. Borel measurable of additive class $\alpha$, has the Baire property) in $X$. We note that, by $0.4(1)$, for

(2) If $\mathrm{P}$ is a property of subsets of a topological space $X$, then $E \subset X$ is locally $\mathrm{P}$ if each $x$ in $E$ has a neighborhood $U$ in $X$ such that $U \cap E$ has property $\mathrm{P}$. 
each of these properties, $f: X \rightarrow Y$ has the property if and only if $f: X \rightarrow f(X)$ has it, and also that each of the properties is preserved by restriction. If $f$ is Borel measurable of class $\alpha$, we sometimes say more briefly that $f$ is "of class $\alpha$." Notice that the mappings of class 0 are precisely the continuous mappings.

A mapping $f: X \rightarrow Y$ is said to be a generalized homeomorphism of class $(\alpha, \beta)$ if $f$ is $1-1, f$ is of class $\alpha$, and $f^{-1}$ is of class $\beta$. A generalized homeomorphism of class $(0,0)$ is a homeomorphism in the usual sense.

The following properties are well known.

(8) If $f: X \rightarrow Y$ is of class $\alpha$ and $B \subset Y$ is a set of class $\beta$, then the set $f^{-1}(B)$ is of class $\alpha+\beta$ (and is multiplicative or additive according to the class of $B$ ) $[6, p$. 376, Theorem 1].

(9) If $f: X \rightarrow Y$ is of class $\alpha$ and $g: Y \rightarrow Z$ is of class $\beta$, then the composite mapping $g \circ f$ is of class $\alpha+\beta[6$, p. 376, Theorem 2].

0.6 Baire space. $B(k)$ is defined [13, p. 5] to be the product space $\Pi T_{n}$ $(n=1,2, \ldots)$, where each $T_{n}$ is a discrete space of cardinal $k . B(k)$ can be completely metrised by the metric $\rho$ for which $\rho(s, t)=1 / n$ if $s_{1}=t_{1}, \ldots, s_{n-1}=t_{n-1}, s_{n} \neq t_{n}$ $(s, t \in B(k))$. We denote by $S(t, n)$ the basic neighborhood of $t$ consisting of all points $s$ such that $s_{1}=t_{1}, \ldots, s_{n}=t_{n}$.

THEOREM (STONE [13, p. 6]). If $X$ is a metrisable, 0-dimensional absolute $G_{\delta}$ with a dense set of cardinal $k$, and if every nonempty open subset of $X$ contains a discrete subset of cardinal $k$, then $X$ is homeomorphic to $B(k)$ and conversely.

An immediate corollary of this theorem is

(10) The discrete union of $\leqq k$ copies of $B(k)$ is homeomorphic to $B(k)$.

(By the "discrete union" of a family of topological spaces $\left\{X_{t}\right\}$ we mean the disjoint union $X$ topologized so that $U \subset X$ is open iff the intersection of $U$ with each $X_{t}$ is open in $X_{t}$ for every $t$.)

1. $\sigma$-discrete decompositions. For some time now the notion of discreteness, as a property of sets or families of sets, together with its derivatives ( $\sigma$-discreteness, etc.) have been among the most fundamental concepts used by topologists in classifying and studying topological spaces. It has been especially adaptable for describing a wide range of topological phenomena common to those spaces which we call metrisable (see e.g. [2] and [14]). Here we introduce and study another derivative of this basic notion, namely that of a " $\sigma$-discrete decomposition" (see $\S 1.2)$, which we believe has not previously been investigated. That this concept too is of fundamental character will become evident in later sections as it applies to the general theory of Borel measurable mappings.

1.1 Discrete collections of sets. We recall that a collection $\left\{A_{d} \mid d \in D\right\}$ of subsets of a (metric) space $X$ is said to be relatively discrete if each point of $\bigcup A_{d}(d \in D)$ has a neighborhood which meets exactly one member of the collection; and that 
this is equivalent to the existence of open sets $U_{d}$ in $X$ such that $A_{d} \subset U_{d}$ and $A_{d^{\prime}} \cap U_{d}=\varnothing$ for $d^{\prime} \neq d\left(d, d^{\prime} \in D\right)$. We say that the collection $\left\{A_{d} \mid d \in D\right\}$ is discrete in $X$ if each point of $X$ has a neighborhood which meets at most one member of the collection; this is equivalent to saying that $\left\{\bar{A}_{d} \mid d \in D\right\}$ is relatively discrete and $\bigcup_{d} \bar{A}_{d}=\mathrm{Cl}\left(\cup_{d} A_{d}\right)$, where the closures are taken in $X$. Finally, we say that $\left\{A_{d} \mid d \in D\right\}$ is metrically discrete (with respect to some metric $\rho$ on $X$ ) if for some $\varepsilon>0$ we have $\rho\left(A_{d}, A_{d^{\prime}}\right) \geqq \varepsilon$ for each pair of distinct elements $d, d^{\prime}$ in $D$; and in this case we say that the collection is $\varepsilon$-discrete.

REMARK. One easily sees that metrically discrete implies discrete and discrete implies relatively discrete. The reverse implications do not hold, even in the case when the members consist of just one point. For example, with the usual metric for the real numbers $R$, the collection $\{\{1 / n\} \mid n=1,2, \ldots\}$ is relatively discrete, but not discrete in $\boldsymbol{R}$; and

$$
\{\{n\} \mid n=1,2, \ldots\} \cup\{\{n+1 / n\} \mid n=1,2, \ldots\}
$$

is discrete in $\boldsymbol{R}$, but not metrically discrete.

$1.2 \sigma$-discrete decomposition. For a given collection $\left\{A_{d} \mid d \in D\right\}$ of subsets of a metric space $X$ we consider the following three properties:

(1) Each $A_{d}=\bigcup\left\{A_{d n} \mid n=1,2, \ldots\right\}(d \in D)$ where $\left\{A_{d n} \mid d \in D\right\}$ is relatively discrete, $n=1,2, \ldots$.

(2) Each $A_{d}=\bigcup\left\{B_{d n} \mid n=1,2, \ldots\right\}(d \in D)$ where $\left\{B_{d n} \mid d \in D\right\}$ is discrete in $X$, $n=1,2, \ldots$

(3) Each $A_{d}=\bigcup\left\{C_{d n} \mid n=1,2, \ldots\right\}(d \in D)$ where $\left\{C_{d n} \mid d \in D\right\}$ is metrically discrete, $n=1,2, \ldots$.

Each of these properties could reasonably be used to define what might be called a " $\sigma$-discrete decomposition" for $\left\{A_{d} \mid d \in D\right\}$. Fortunately, however, it does not matter which we use in view of the following lemma.

LEMMA 2. Properties (1), (2), and (3) above are all equivalent.

Proof. We have already observed that (3) implies (2) and that (2) implies (1) trivially; hence we need only prove that (1) implies (3).

Suppose $\left\{A_{d} \mid d \in D\right\}$ satisfies (1). Then for each $A_{d n}$ we can define sets

$$
C_{d n m}=\left\{x \in A_{d n} \mid \rho\left(x, A_{d^{\prime} n}\right) \geqq 1 / m \text { for all } d^{\prime} \neq d\right\},
$$

$m=1,2, \ldots$ Since each $x$ in $A_{d n}$ is the center of some ball which meets only $A_{d n}$, it follows that $x$ belongs to some $C_{d n m}$; i.e., we have $A_{d n}=\bigcup\left\{C_{d n m} \mid m=1,2, \ldots\right\}$. Moreover, since $\rho\left(x, A_{d^{\prime} n}\right) \geqq 1 / m$ for each $x$ in $C_{d n m}\left(d^{\prime} \neq d\right)$ and $C_{d^{\prime} n m} \subset A_{d^{\prime} n}$, it is clear that the collection $\left\{C_{d n m} \mid d \in D\right\}$ is $1 / m$-discrete for each $n=1,2, \ldots$ Thus, we need only write the double sequence $(n, m)$ as a simple one to satisfy property (3).

1.3 Remark. Property (1) depends only on the collection $\left\{A_{d} \mid d \in D\right\}$ itself and is a topological invariant (in the sense that if $h$ is an embedding of $\bigcup\left\{A_{d} \mid d \in D\right\}$, 
then the collection $\left\{h\left(A_{d}\right) \mid d \in D\right\}$ also has this property). Thus, as a corollary of Lemma 2, a collection which has these properties for any one containing space $X$ has them for all $X$. Such a collection will be said to have a $\sigma$-discrete decomposition or to be $\sigma$-discretely decomposable; both abbreviated $\sigma$-d.d. (Compare the comments in $[14, \S 2.1]$.)

1.4 Some lemmas on $\sigma$-discrete decompositions. If a given collection $\left\{A_{d} \mid d \in D\right\}$ is $\sigma$-d.d., clearly so is every collection of the form $\left\{B_{d} \mid d \in D\right\}$ where $B_{d} \subset A_{d}$ for each $d$ in $D$.

LEMMA 3. Let $\left\{A_{d} \mid d \in D\right\}$ be a collection of subsets of a metric space $X$. If there exists a $\sigma$-discrete cover $\mathscr{B}$ of $\bigcup\left\{A_{d} \mid d \in D\right\}$ by subsets $B$ of $X$ such that each of the collections $\left\{A_{d} \cap B \mid d \in D\right\}$ is $\sigma$-discretely decomposable, then $\left\{A_{d} \mid d \in D\right\}$ is $\sigma$-discretely decomposable.

Proof. In view of Remark 1.3, there is no loss in generality in assuming that $X=\bigcup\left\{A_{d} \mid d \in D\right\}$. Thus, by hypothesis, $\mathscr{B}=\bigcup\left\{B_{t m} \mid t \in T_{m}\right\}(m=1,2, \ldots)$, where the subcollections obtained by fixing $m$ are discrete in $X$. Furthermore, for fixed $m$ and $t$ in $T_{m}$, each

$$
A_{d} \cap B_{t m}=\bigcup\left\{A_{d t m n} \mid n=1,2, \ldots\right\} \quad(d \in D)
$$

where the subcollections obtained by fixing $n$ are discrete in $X$. Put $A_{d m n}$ $=\bigcup\left\{A_{d t m n} \mid t \in T_{m}\right\}$ and observe that we have the following chain of equalities:

$$
\begin{aligned}
\bigcup_{m} \bigcup_{n} A_{d m n} & =\bigcup_{m} \bigcup_{n} \bigcup_{t \in T_{m}} A_{d t m n}=\bigcup_{m} \bigcup_{t \in T_{m}} \bigcup_{n} A_{d t m n}=\bigcup_{m} \bigcup_{t \in T_{m}} A_{d} \cap B_{t m} \\
& =A_{d} \cap\left(\bigcup_{m} \bigcup_{t \in T_{m}} B_{t m}\right)=A_{d} \cap(\bigcup \mathscr{B})=A_{d} .
\end{aligned}
$$

Thus, if for fixed $m$ and $n$ we can show that the collection $\left\{A_{d m n} \mid d \in D\right\}$ is discrete in $X$, then it will follow that $\left\{A_{d} \mid d \in D\right\}$ is $\sigma$-d.d.

Fixing $m$ and $n$, for a given $x$ in $X$, we can find a neighborhood of $x, U$ say, such that $U$ meets at most one of the sets $B_{t m}\left(t \in T_{m}\right)$. If $U$ meets no $B_{t m}$, then clearly $U$ meets no $A_{d m n}$. Thus suppose $U$ meets some $B_{t m}$, say $B_{t^{\prime} m}$. By the above remarks, there exists a neighborhood $V$ of $x$ such that $V$ meets at most one $A_{d t^{\prime} m n}$, and, accordingly, $U \cap V$ is a neighborhood of $x$ which meets at most one of the sets $A_{d m n}$. This proves that the sets $A_{d m n}$ (for fixed $m$ and $n$ ) form a discrete collection, and completes the proof of the lemma.

Definition. We say that $\left\{A_{d} \mid d \in D\right\}$ is locally $\sigma$-d.d. if each point of $\bigcup A_{d}$ has a neighborhood $U$ such that $\left\{U \cap A_{d} \mid d \in D\right\}$ is $\sigma$-d.d.

COROllary 1. A locally $\sigma-d . d$. collection in a metric space $X$ is $\sigma$-d.d.

Proof. If $\left\{A_{d} \mid d \in D\right\}$ is such a collection, then we can find an open cover $\left\{U_{s}\right\}$ of $\bigcup A_{d}$ such that each $\left\{U_{s} \cap A_{d} \mid d \in D\right\}$ is $\sigma$-d.d. By the paracompactness of $X$, there exists a $\sigma$-discrete open refinement $\mathscr{B}$ of $\left\{U_{s}\right\}$. Moreover, since each $B$ in $\mathscr{B}$ is $\subset$ some $U_{s}$, the collection $\left\{B \cap A_{d} \mid d \in D\right\}$ is $\sigma$-d.d. Lemma 3 now applies and says that $\left\{A_{d} \mid d \in D\right\}$ is $\sigma$-d.d. 
LEMMA 4. If $\left\{A_{d} \mid d \in D\right\}$ is a $\sigma$-d.d. collection of subsets of a metric space $X$, then there exist metrically discrete open collections $\left\{U_{d n} \mid d \in D\right\}(n=1,2, \ldots)$ such that $A_{d} \subset \bigcup_{n=1}^{\infty} U_{d n}$ for each $d$ in $D$.

Proof. Assuming the hypothesis, there exists by Lemma $2(\$ 1.2)$ metrically discrete collections $\left\{C_{d n} \mid d \in D\right\}(n=1,2, \ldots)$ such that $A_{d}=\bigcup C_{d n}(n=1,2, \ldots)$. Suppose $\rho\left(C_{d n}, C_{d^{\prime} n}\right) \geqq \varepsilon_{n}>0$ for $d \neq d^{\prime}(n=1,2, \ldots)$. Let

$$
U_{d n}=\left\{x \mid \rho\left(x, C_{d n}\right)<\varepsilon_{n} / 3\right\} .
$$

Then $\left\{U_{d n} \mid d \in D\right\}$ is easily seen to be $\varepsilon_{n} / 3$-discrete. Moreover, we have $A_{d}$ $=\bigcup_{n} C_{d n} \subset \bigcup_{n} U_{d n}$; completing the proof.

LEMMA 5. If $f$ is a continuous map of a metric space $X$ onto a metric space $Y$ and $\left\{A_{d} \mid d \in D\right\}$ is a $\sigma$-d.d. collection of subsets of $Y$, then $\left\{f^{-1}\left(A_{d}\right) \mid d \in D\right\}$ is $\sigma$-d.d.

Proof. Suppose $A_{d}=\bigcup\left\{A_{d n} \mid n=1,2, \ldots\right\}$ where for fixed $n$ the sets $A_{d n}$ form a discrete collection in $Y$. Since $f^{-1}\left(A_{d}\right)=\bigcup_{n} f^{-1}\left(A_{d n}\right)$, it suffices to show that, for fixed $n$, the sets $f^{-1}\left(A_{d n}\right)$ form a discrete collection in $X$. But, given $x$ in $X$, there exists an open set $V$ containing $f(x)$ and meeting at most one $A_{d n}$; hence, by continuity of $f, f^{-1}(V)$ is an open set in $X$, contains $x$, and meets at most one $f^{-1}\left(A_{d n}\right)$ as required.

LEMMA 6. A collection $\left\{A_{d} \mid d \in D\right\}$ with $\left\|A_{d}\right\|=1$ for all $d$ in $D$ is $\sigma-d . d$. if and only if $\bigcup_{d} A_{d}$ is $\sigma$-discrete.

Proof. Suppose $A_{d}=\bigcup\left\{A_{d n} \mid n=1,2, \ldots\right\}$, where for fixed $n$ the sets $A_{d n}$ form a discrete collection in $\bigcup_{d} A_{d}$. Then $\left\|A_{d n}\right\| \leqq 1$ for each $d$ in $D$ and hence $\bigcup_{d} A_{d n}$ is a discrete subset of $\bigcup A_{d}$. Since $\bigcup_{d} A_{d}=\bigcup_{n} \bigcup_{d} A_{d n}$, it follows that $\bigcup_{d} A_{d}$ is $\sigma$ discrete.

Conversely, if $\bigcup_{d} A_{d}=\bigcup_{n} B_{n}$ where each $B_{n}$ is a discrete set, then $A_{d}$ $=\bigcup\left\{A_{d} \cap B_{n} \mid n=1,2, \ldots\right\}$ and for fixed $n$ the sets $A_{d} \cap B_{n}$ clearly form a discrete collection. Thus the original collection is $\sigma$-d.d.

LEMMA 7. If $\left\{A_{d} \mid d \in D\right\}$ is a $\sigma-d . d$. collection of sets which are analytic (resp. of additive class $\alpha$ ) in some fixed metric space $X$, then $\bigcup\left\{A_{d} \mid d \in D^{\prime}\right\}$ is analytic (of additive class $\alpha$ ) in $X$ for all $D^{\prime} \subset D$.

Proof. We will prove the lemma for analytic sets; the proof of the other case is entirely analogous.

By Lemma 4, there exists for each $n=1,2, \ldots$ an open discrete collection $\left\{U_{d n} \mid d \in D\right\}$ such that $A_{d} \subset \bigcup_{n} U_{d n}$ for each $d$ in $D$. Now given $D^{\prime} \subset D$, the sets $U_{d n} \cap A_{d}$ for $d$ in $D^{\prime}$ form a discrete collection of analytic sets in $X$, and so its union is analytic in $X$ by $0.4(5)$. Hence $\bigcup\left\{A_{d} \mid d \in D^{\prime}\right\}=\bigcup_{n} \bigcup\left\{U_{d n} \cap A_{d} \mid d \in D^{\prime}\right\}$ is also analytic in $X$ by $0.4(3)$, proving the lemma. 
LEMMA 8. If $\left\{A_{d} \mid d \in D\right\}$ is non- $\sigma$-discretely decomposable, then there exists a non- $\sigma$-discrete subset $F$ of $\bigcup A_{d}$ at each point of which the collection fails to be locally $\sigma-d . d$.

Proof. For let $G$ be the set of all points in $\bigcup A_{d}$ at which the collection is $\sigma$-d.d. Then, by Corollary 1 , it follows that $\left\{A_{d} \cap G \mid d \in D\right\}$ is $\sigma$-d.d. Now let $F=\bigcup A_{d}$ $\sim G$. Then, since $F$ and $G$ together form a finite and therefore $\sigma$-discrete cover for $\bigcup A_{d}$, it follows from Lemma 3 that $\left\{F \cap A_{d} \mid d \in D\right\}$ is not $\sigma$-d.d., and hence, in particular that $F$ is not $\sigma$-discrete (see the proof of Lemma 6).

We will need in particular the following corollary.

Corollary 2. If $\left\{A_{d} \mid d \in D_{i}\right\}(i=0,1)$ are two non- $\sigma$-d.d. collections of subsets of a metric space $X$, then there exist, for each natural number $n$, open sets $V_{i}$ in $X$ such that the diameter of $V_{i}$ is $\leqq 1 / n, \bar{V}_{0} \cap \bar{V}_{1}$ is empty, and $\left\{A_{d} \cap V_{i} \mid d \in D_{i}\right\}$ is non- $\sigma$-d.d., $i=0,1$.

Proof. Since countable sets are $\sigma$-discrete, it follows that the set $F$ of Lemma 8 is uncountable; in particular it contains more than one point and hence we can choose distinct points $x_{i}$ in $\bigcup\left\{A_{d} \mid d \in D_{i}\right\}$ such that every neighborhood of $x_{i}$ meets the collection in a non- $\sigma$-d.d. collection. Hence, if the distance between $x_{0}$ and $x_{1}$ is denoted by $s$, then we may take $V_{i}$ to be the ball which is centered at $x_{i}$ and with radius $\min (1 / n, s / 3)$, and the above-mentioned properties are at once verified.

\section{The basic theorem.}

2.1. If $\mathbf{P}$ denotes a topological property (that is, a class of topological spaces), then a collection of subsets of a fixed space will be said to be "completely additive$\mathrm{P}$ " or a "completely additive collection of $\mathrm{P}$ sets" provided the union of every subcollection, as a topological space, has property P. In this section we are concerned mainly with the study of disjoint collections of analytic sets (contained in some fixed metric space $X$ ) which are completely additive-absolutely analytic. Such collections arise naturally in the study of Borel measurable and analytic mappings: if $X$ is complete and $f: X \rightarrow Y$ is Borel measurable (or analytic), and if $\left\{V_{d} \mid d \in D\right\}$ is a disjoint collection of open subsets of $Y$, then $\left\{f^{-1}\left(V_{d}\right) \mid d \in D\right\}$ is disjoint and completely additive-absolutely analytic. Our principal result concerning these collections is Theorem $2(\$ 2.7)$ which says that they are always $\sigma$-discretely decomposable. This result provides the foundation upon which we base our subsequent study of the properties of Borel measurable mappings with a nonseparable range. We begin by considering first some special cases.

For separable $X$ we have the following theorem.

2.2 THEOREM 1. On the continuum hypothesis, a disjoint collection of analytic subsets of a separable metric space $X$ is completely additive-analytic in $X$ if and only if it is countable. 
Proof. That a countable collection of analytic sets is completely additiveanalytic follows from $0.4(3)$.

Conversely, suppose we have a completely additive collection of analytic sets in a separable metric space $X$, and suppose this collection is uncountable. Then, by taking unions of all possible subcollections, $X$ must have $\geqq 2 \aleph_{1}$ analytic subsets; and, on the continuum hypothesis, $2^{\aleph_{1}}=2^{c}>c$. But this contradicts $0.4(6)$; hence the original collection must be countable, proving the theorem.

2.3 Remarks. We will see later (2.8, Corollary 7) that Theorem 1 continues to hold without the continuum hypothesis, provided we assume $X$ is absolutely analytic. Since any discrete collection of analytic sets is completely additiveanalytic by $0.4(5)$, the above theorem is false in the absence of separability. Thus countability does not serve to characterize these collections; nor does discreteness as trivial (two membered) examples show.

Let us consider another special case, namely those collections whose members consist of a single point. Further, let us assume that the union of every subcollection is absolutely Borel. Evidently, this is equivalent to the study of absolute Borel sets all of whose subsets are Borel. Such a study has been made by A. H. Stone (see [14] for eight equivalent properties) and, in particular, he has shown that such a set must be $\sigma$-discrete. This latter property also characterizes the absolutely analytic sets all of whose subsets are analytic (see $\$ 2.8$, Corollary 4).

These considerations lead one to suspect that possibly $\sigma$-discreteness (as a property of collections of sets [5, p. 127]) is characteristic of disjoint completely additive collections of absolutely analytic sets. That this property is sufficient for complete additivity follows from $0.4(3)$ and $0.4(5)$. It is not, however, necessary, as the following example shows.

2.4 EXAmple. Consider the Baire space

$$
B\left(\aleph_{1}\right)=\prod T_{n} \quad(n=1,2, \ldots)
$$

where each $T_{n}$ is a copy of the set of all countable ordinals. For convenience of notation let us write $t(\beta n \alpha)$ for the point of this space whose first $n$ coordinates are all equal to $\beta$ and whose remaining coordinates are all equal to $\alpha$. Also, we will denote by $t(\beta)$ that point which has all its coordinates equal to $\beta$. We now define, for each countable ordinal $\alpha$, sets $A_{\alpha}=\{t(\beta n \alpha) \mid \beta \geqq \alpha$ and $n=1,2, \ldots\}$. It is immediate that the sets $A_{\alpha}$ are pairwise disjoint. Also, since $t(\beta n \alpha) \rightarrow t(\beta)$ as $n \rightarrow \infty$, it follows that $t(\beta) \in \bar{A}_{\alpha} \cap A_{\beta}$ whenever $\alpha<\beta$. This last property implies that the only discrete subcollections of the collection $\left\{A_{\alpha}\right\}$ are the ones with at most one member. Since the entire collection is uncountable, it follows that it cannot be $\sigma$-discrete. However, the set $A=\bigcup A_{\alpha}$ is a $\sigma$-discrete set, hence every subset is an absolute $F_{\sigma}$-set [14, Theorem 2]. To prove this, for each $n$ let $D_{n}$ $=\{t(\beta n \alpha) \mid \alpha$ and $\beta$ are ordinals and $\beta \geqq \alpha\}$. For each $x$ in $B\left(\aleph_{1}\right)$, we clearly have $\left\|S(x, n+1) \cap D_{n}\right\| \leqq 1$. Hence $D_{n}$ is discrete. Moreover, $A=\bigcup_{n=1}^{\infty} D_{n}$, proving 
that $A$ is $\sigma$-discrete $\left({ }^{3}\right)$. It follows that the sets $A_{\alpha}\left(\alpha<\omega_{1}\right)$ form a disjoint completely additive collection of absolute $F_{\sigma}$-sets which is not $\sigma$-discrete.

RemarK. The sets $A_{\alpha}$, in the above example, do however form a $\sigma$-discretely decomposable collection: for if $A=\bigcup D_{n}(n=1,2, \ldots)$, where each $D_{n}$ is discrete, then one easily checks that $\left\{A_{\alpha} \cap D_{n} \mid \alpha<\omega_{1}, n=1,2, \ldots\right\}$ is the required $\sigma$-discrete decomposition. We have already seen (Lemma $7, \S 1.4)$ that a $\sigma$-d.d. collection of analytic sets is completely additive (-analytic). We now shall prove that the converse is true for completely additive-absolutely analytic collections.

2.5 The basic lemma. We require first a lemma.

LemMa 9. If $\left\{A_{d} \mid d \in D\right\}$ is a disjoint collection of analytic sets in a complete metric space $X$ which is non- $\sigma$-discretely decomposable, then $D$ can be partitioned into two disjoint subsets $D_{i}$ such that $\left\{A_{d} \mid d \in D_{i}\right\}$ is non- $\sigma$-discretely decomposable, $i=0,1$.

Proof. We define inductively a sequence of open sets $V_{p}$ and positive integers $n_{p}(p=0,1,2, \ldots)$ subject to the following conditions:

$\left(1_{p}\right) \delta\left(V_{p}\right) \leqq 1 /(p+1)$,

(2p) $V_{p} \subset V_{p-1}$ (where $\left.V_{-1}=X\right)$,

(3) $\left\{A_{n_{1} \ldots n_{p}}^{d} \cap V_{p} \mid d \in D\right\}$ is non- $\sigma$-d.d.

(Here $\delta(E)$ denotes the diameter of $E \subset X$ [with respect to some fixed metric on $X$ ], and the meaning of $A_{n_{1} \ldots n_{p}}^{d}$ is that given in Lemma 1 of $\$ 0.1$ applied to the analytic sets $A_{d}$.)

We may, of course, assume that $\delta(X) \leqq 1$, so that, putting $V_{0}=X, n_{0}=0$, and $A^{d}=A_{d}$, properties $\left(1_{0}\right),\left(2_{0}\right)$, and $\left(3_{0}\right)$ hold by hypothesis. Now assume that open sets $V_{p}$ and positive integers $n_{p}$ have been defined so that $\left(1_{p}\right),\left(2_{p}\right)$, and $\left(3_{p}\right)$ are satisfied, for some integer $p \geqq 0$. By the paracompactness of $X$, we can cover $\bigcup\left\{A_{n_{1} \ldots n_{p}}^{d} \cap V_{p} \mid d \in D\right\}$ by a $\sigma$-discrete open collection of sets with diameters $\leqq 1 /(p+2)$. By Lemma $3(\S 1.4)$, one of these open sets, $V$ say, must be such that the collection $\left\{A_{n_{1} \ldots n_{p}}^{d} \cap V_{p} \cap V \mid d \in D\right\}$ is non- $\sigma$-d.d. Accordingly, we set $V_{p+1}$ $=V_{p} \cap V$. Now, observe that the sets $\bigcup\left\{A_{n_{1} \ldots n_{p} n}^{d} \mid d \in D\right\}(n=1,2, \ldots)$ form a countable and therefore $\sigma$-discrete cover for $\bigcup\left\{A_{n_{1} \ldots n_{p}}^{d} \cap V_{p+1} \mid d \in D\right\}$. Thus, again by Lemma 3, there exists a suffix $n_{p+1}$ such that the sets

$$
\left(A_{n_{1} \ldots n_{p}}^{d} \cap V_{p+1}\right) \cap \bigcup_{d^{\prime} \in D} A_{n_{1} \ldots n_{p} n_{p+1}}^{d^{\prime}}=A_{n_{1} \ldots n_{p+1}}^{d} \cap V_{p+1}
$$

as $d$ ranges over $D$, form a non- $\sigma$-d.d. collection. Hence we have shown that properties $\left(1_{p+1}\right)-\left(3_{p+1}\right)$ are satisfied for these choices of $V_{p+1}$ and $n_{p+1}$. Thus open sets $V_{p}$ and positive integers $n_{p}$ can be defined by induction for every $p \geqq 0$.

$\left({ }^{3}\right)$ This proof was supplied by the referee, and is simpler and much more direct than the author's original proof. 
Now let $I_{p}=\left\{d \in D \mid A_{n_{1} \ldots n_{p}}^{d} \cap V_{p} \neq \varnothing\right\}, \quad p=0,1, \ldots$, and put $I_{\infty}=\bigcap I_{p}$ $(p=0,1, \ldots)$. Since the collection consisting of just the empty set has a trivial $\sigma$-discrete decomposition, it follows from $\left(3_{p}\right)$ that $I_{p} \neq \varnothing$ for all $p$. We shall now show that $\left\|I_{\infty}\right\| \leqq 1$. For suppose $d_{0}$ and $d_{1}$ both belong to $I_{\infty}$. Then, for $j=0,1$, the collection $\left\{\bar{A}_{n_{1} \ldots n_{p}}^{d_{j}} \cap \bar{V}_{p} \mid p=0,1, \ldots\right\}$ is a nested sequence of nonempty closed subsets of $X$ with diameters converging to zero. The space $X$ being complete, the intersection of this system of sets must consist of a single point $x_{j}(j=0,1)$. Since both points belong to $\bigcap \bar{V}_{p}(p=0,1, \ldots)$ and the diameters of the sets $\bar{V}_{p}$ converge to zero by $\left(1_{p}\right)$, we must have $x_{0}=x_{1}$. On the other hand,

$$
x_{j} \in \bigcap\left\{\bar{A}_{n_{1} \ldots n_{p}}^{d_{j}} \mid p=0,1, \ldots\right\} \subset A_{d_{j}} \text { and } A_{d_{0}} \cap A_{d_{1}}=\varnothing
$$

whenever $d_{0} \neq d_{1}$. Hence we must have $d_{0}=d_{1}$, which proves that $\left\|I_{\infty}\right\| \leqq 1$.

Now the sets $\bigcup\left\{A_{d} \mid d \in I_{p} \sim I_{p+1}\right\}(p=0,1, \ldots)$ together with $\bigcup\left\{A_{d} \mid d \in I_{\infty}\right\}$ form a countable and therefore $\sigma$-discrete cover for $\bigcup\left\{A_{d} \mid d \in D\right\}$. Hence, from Lemma $3(\S 1.4)$ and the fact that $\left\{A_{d} \mid d \in I_{\infty}\right\}$ is $\sigma$-d.d. (since $\left\|I_{\infty}\right\| \leqq 1$ ), it follows that for some $q$ the collection of sets $A_{d}$ as $d$ ranges over $I_{q} \sim I_{q+1}$ is non- $\sigma$-d.d. Since $\left\{A_{d} \mid d \in I_{q+1}\right\}$ is non- $\sigma$-d.d. by $\left(3_{q+1}\right)$, the lemma follows upon taking $D_{0}=I_{q+1}$ and $D_{1}=D \sim I_{q+1}$.

2.6 REMARK. As a corollary to our method of proof we have: if $\left\|A_{d}\right\|=1$ for all $d$ in $D$ and $\bigcup A_{d}(d \in D)$ is analytic in $X$, then $\bigcup\left\{A_{d} \mid d \in D_{i}\right\}$ is analytic in $X$, $i=0,1$. This follows from the fact that

$$
\begin{aligned}
\bigcup\left\{A_{d} \mid d \in D_{0}\right\} & =\bigcup\left\{A_{d} \mid d \in I_{q+1}\right\} \\
& =\bigcup\left\{A_{d} \mid A_{d} \cap V_{q+1} \neq \varnothing\right\}=\left(\bigcup\left\{A_{d} \mid d \in D\right\}\right) \cap V_{q+1}
\end{aligned}
$$

(which is clearly analytic in $X$ ); and, consequently, $\bigcup\left\{A_{d} \mid d \in D_{1}\right\}=\bigcup_{d \in D} A_{d}$ $\cap\left(X \sim V_{q+1}\right)$ (and hence also analytic in $\left.X\right)$.

\subsection{The basic theorem.}

THEOREM 2. A disjoint collection of analytic sets in a complete metric space $X$ is completely additive-analytic (in $X$ ) if and only if it is $\sigma$-d.d.

Proof. In view of Lemma $7(\S 1.4)$ we have only to prove the necessity. The proof will be by contradiction, and we assume $\left\{A_{d} \mid d \in D\right\}$ is a disjoint completely additive-analytic collection of sets in $X$ which is non- $\sigma$-d.d. The proof now rests upon the construction of a Cantor set $C \subset \bigcup\left\{A_{d} \mid d \in D\right\}$ with the property that $\left\|C \cap A_{d}\right\| \leqq 1$ for every $d \in D$.

We begin by partitioning $D$ into two disjoint subsets $D_{i(1)}$ such that $\left\{A_{d} \mid d \in D_{i(1)}\right\}$ is non- $\sigma$-d.d., $i(1)=0,1$. This we can do by the preceding lemma. By Corollary 2 of Lemma $8(\$ 1.4)$, we can find open sets $V_{i(1)}$ such that $\left\{A_{d} \cap V_{i(1)} \mid d \in D_{i(1)}\right\}$ is non- $\sigma$-discretely decomposable, $\delta\left(V_{i(1)}\right) \leqq 1$, and $\bar{V}_{0} \cap \bar{V}_{1}$ $=\varnothing$. Now consider

$$
A^{i(1)}=\bigcup\left\{A_{d} \cap V_{i(1)} \mid d \in D_{i(1)}\right\}
$$


As follows easily from the fact that the $A_{d}$ 's are completely additive-analytic, the set $A^{i(1)}$ is analytic in $X$. Hence, applying Lemma $3(\S 1.4)$ to the countable cover $\left\{A_{1}^{i(1)}, A_{2}^{i(1)}, \ldots\right\}$ of $A^{i(1)}$ (in the notation of Lemma 1, $\S 0.1$ ), we deduce the existence of a suffix $n_{1}^{i(1)}$ such that

$$
\left\{A_{d} \cap A_{n_{1}^{i(1)}}^{i(1)} \mid d \in D_{i(1)}\right\}
$$

is non- $\sigma$-d.d., disjoint, and completely additive-analytic in $X$. We now put $A_{d}^{i(1)}$ $=A_{d} \cap A_{n_{1}^{(1)}(1)}^{i\left(\subset V_{i(1)}\right)}$ for each $d \in D_{i(1)}$.

Now assume that for each natural number $p \leqq q$ and for each $p$-tuple $i(p)$ $=\left(i_{1}, \ldots, i_{p}\right)$ where $i_{r}=0$ or $1, r=1, \ldots, p$ (i.e., for each "dyadic" $p$-tuple), we have defined open sets $V_{i(p)} \subset X$, sets $D_{i(p)} \subset D$, sets $A^{i(p)}$ and $A_{d}^{i(p)}\left(d \in D_{i(p)}\right)$ analytic in $X$, and natural numbers $n_{1}^{i(p)}, \ldots, n_{p}^{i(p)}$ subject to the following:

$\left(4_{p}\right) \delta\left(V_{i(p)}\right) \leqq 1 / p$,

(5) $\bar{V}_{i(p-1), 0} \cap \bar{V}_{i(p-1), 1}=\varnothing$ and $D_{i(p-1), 0} \cap D_{i(p-1), 1}=\varnothing$,

$\left(6_{p}\right) A^{i(p)}=\bigcup\left\{A_{d}^{i(p-1)} \cap V_{i(p)} \mid d \in D_{i(p)}\right\} \neq \varnothing$,

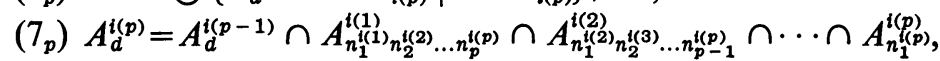

$\left(8_{p}\right)\left\{A_{d}^{i(p)} \mid d \in D_{i(p)}\right\}$ is non- $\sigma$-d.d., disjoint, and completely additive-analytic in $X$.

(Here we make the conventions that $i(p-1), j=\left(i_{1}, \ldots, i_{p-1}, j\right), V_{i(0), j}=V_{j}$, and $A_{d}^{i(0)}=A_{d}$.)

We have already seen that the sets $V_{i(1)}, D_{i(1)}, A^{i(1)}$, and $A_{d}^{i(1)}$ and the numbers $n_{1}^{i(1)}$ defined above satisfy properties $\left(4_{1}\right)-\left(8_{1}\right)$. Now let $i(q)$ be a given dyadic $q$-tuple. By $\left(8_{q}\right)$ and Lemma $9(\S 2.5)$, we can partition $D_{i(q)}$ into two disjoint subsets $D_{i(q+1)}$ $(i(q+1)=i(q), 0$ and $i(q), 1)$ such that $\left\{A_{d}^{i(q)} \mid d \in D_{i(q+1)}\right\}$ is non- $\sigma$-d.d. By Corollary 2 of Lemma $8(\$ 1.4)$, we can then find open sets $V_{i(q+1)}$ with $\delta\left(V_{i(q+1)}\right) \leqq 1 /(q+1)$ such that

$$
\left\{A_{d}^{i(q)} \cap V_{i(q+1)} \mid d \in D_{i(q+1)}\right\}
$$

is non- $\sigma$-d.d. and $\bar{V}_{i(q), 0} \cap \bar{V}_{i(q), 1}=\varnothing$. It follows that

$$
A^{i(q+1)}=\bigcup\left\{A_{d}^{i(q)} \cap V_{i(q+1)} \mid d \in D_{i(q+1)}\right\}
$$

is nonempty and, by $\left(8_{q}\right)$, analytic in $X$. Now consider the collection of sets $A^{i(q+1)}\left[n_{1} \cdots n_{q+1}\right]$ obtained from the formula

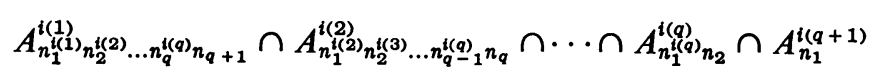

where $n_{r}$ can take on all positive integral values, $r=1, \ldots, q+1$. By property $\left(7_{q}\right)$ and Lemma 1 (\$0.1), these sets constitute a countable and hence $\sigma$-discrete cover of $A^{i(q+1)}$. Consequently, by Lemma $3(\S 1.4)$, there exist suffixes $n_{1}^{i(q+1)}, \ldots, n_{q+1}^{i(q+1)}$ such that

$$
\left\{A_{d}^{i(q)} \cap A^{i(q+1)}\left[n_{1}^{i(q+1)} \cdots n_{q+1}^{i(q+1)}\right] \mid d \in D_{i(q+1)}\right\}
$$


is non- $\sigma$-d.d. Also, clearly, this collection is disjoint and, by $\left(8_{q}\right)$, completely additive-analytic in $X$. Moreover, if we set, for each $d \in D_{i(q+1)}$,

$$
A_{d}^{i(q+1)}=A_{d}^{i(q)} \cap A^{i(q+1)}\left[n_{1}^{i(q+1)} \cdots n_{q+1}^{i(q+1)}\right],
$$

then it is not hard to see from the definitions that $V_{i(q+1)}, D_{i(q+1)}, A^{i(q+1)}$, and $A_{d}^{i(q+1)}$ and $n_{1}^{i(q+1)}, \ldots, n_{q+1}^{i(q+1)}$ satisfy all of the properties $\left(4_{q+1}\right)-\left(8_{q+1}\right)$. Hence we can define by induction the above described sets and natural numbers for every $p$ and for every $p$-tuple $i(p)$, such that $\left(4_{p}\right)-\left(8_{p}\right)$ all hold (where $p=1,2, \ldots$ and the "coordinates" of $i(p)$ assume only the values 0 and 1$)$.

We are now in a position to construct the desired Cantor set. For each dyadic sequence $i=\left(i_{1}, \ldots, i_{p}, \ldots\right)$ (i.e., sequence of 0 's and 1's), with initial $p$-section $i(p)=\left(i_{1}, \ldots, i_{p}\right)$, note that we have defined a sequence

$$
\bar{A}^{i(1)} \supset \bar{A}^{i(2)} \supset \ldots \supset \bar{A}^{i(p)} \supset \ldots
$$

of closed, nonempty (by $\left.\left(6_{p}\right)\right)$ subsets of $X$ with $\delta\left(\bar{A}^{i(p)}\right) \leqq 1 / p$. Also, if $j$ denotes another dyadic sequence, then, by $\left(5_{p}\right)$ and $\left(6_{p}\right), \bar{A}^{i(p)} \cap \bar{A}^{j(p)}=\varnothing$ whenever $i(p)$ $\neq j(p)$. Accordingly, by a well-known theorem $[4, \mathrm{p} .154]$ the subspace $C=\bigcup_{i} \bigcap_{p=1}^{\infty} \bar{A}^{i(p)}$ of $X$ (where the union is over all dyadic sequences) is homeomorphic to the Cantor discontinuum. It remains to show that $C \subset \bigcup\left\{A_{d} \mid d \in D\right\}$ and that $\left\|C \cap A_{d}\right\| \leqq 1$ for all $d \in D$. To prove the first relation, let $i$ be a given dyadic sequence and note that $\left(6_{p+q}\right)$ and $\left(7_{p-1+q}\right)$ imply that

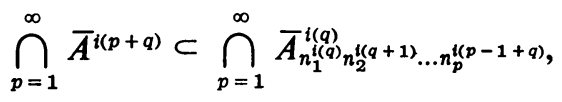

and the latter set is $\subset A^{i(q)}$ by Lemma 1 of $\S 0.1$. Since clearly $A^{i(q)} \subset \bigcup\left\{A_{d} \mid d \in D\right\}$, the first relation is proved. To prove that $C$ meets each $A_{d}$ in at most one point, we note first that the points of $C$ are precisely the intersections $\bigcap \bar{A}^{i(p)}$ $(p=1,2, \ldots)$. Now we have just shown that $\bigcap_{p=1}^{\infty} \bar{A}^{i(p)} \subset A^{i(q)}$ for $q=1,2, \ldots$, and, by $\left(6_{q}\right)$, we have $A^{i(q)} \subset \bigcup\left\{A_{d} \mid d \in D_{i(q)}\right\}$. But, if $i$ and $j$ denote different dyadic sequences, then for some $q$ we have $i(q) \neq j(q)$, and hence $D_{i(q)}$ and $D_{j(q)}$ are disjoint by $\left(5_{q}\right)$; consequently, since the sets $A_{d}$ are pairwise disjoint, the points of $C$ corresponding to the sequences $i$ and $j$ respectively must belong to different $A_{d}$ 's. It follows that $\left\|C \cap A_{d}\right\| \leqq 1$ for all $d$ in $D$.

This leads to a contradiction: for then every subset $S$ of $C$ is of the form $C \cap\left(\bigcup\left\{A_{d} \mid d \in D_{S}\right\}\right)$, for some $D_{S} \subset D$; and such sets, as the intersection of two analytic sets in $X$, are analytic in $X$ and hence in $C(0.4(1))$. But this implies that $C$ has $2^{c}$ analytic subsets contradicting $0.4(6)$. The theorem is thus proved.

REMARK. Theorem 2 will usually be applied when the (metric) space $X$ is absolutely analytic (or absolutely Borel) rather than complete. This is possible since then any collection of subsets of $X$ which is completely additive-analytic in $X$ is also completely additive-analytic in $\tilde{X}$ (the completion of $X$ ). 
2.8 Corollaries to the basic theorem. The assumption of complete additivity in the hypothesis of Theorem 2 is used only to ensure that the sets $A^{i(p)}$ of $\left(6_{p}\right)$ are analytic in $X$. When $\left\|A_{d}\right\|=1$ for all $d$ in $D$ and $\bigcup\left\{A_{d} \mid d \in D\right\}$ is analytic in $X$, it is a consequence of Remark 2.6 and our method of proof that the sets $A^{i(p)}$ are analytic without assuming complete additivity. Thus we have the following theorems of A. H. Stone [14] and A. G. El'kin [3] as consequences of our method of proof.

COROllaRy 3 [STONE (RESP. EL'KIN)]. For each absolute Borel (resp. absolutely analytic) space $X$, one and only one of the following alternatives is true: either (i) $X$ is $\sigma$-discrete, or (ii) X contains a subset homeomorphic to the Cantor set $C\left(\aleph_{0}\right)$.

Corollary 4 (STONE [14]). Every subset of an arbitrary metric space $X$ is an absolute Borel (or absolutely analytic) set if and only if $X$ is $\sigma$-discrete.

Proof. This is a direct consequence of the theorem and Lemma $6(\S 1.4)$ in the case when each $A_{d}$ is a single point.

COROLlaRY 5. If a disjoint collection of absolute Borel sets of additive class $\alpha>0$ is completely additive-absolutely analytic, then it is completely additive-absolutely Borel of additive class $\alpha$.

Proof. This corollary is an immediate consequence of the theorem and Lemma 7 (\$1.4).

COROLlaRY 6. If $X$ is absolutely analytic and of weight $\leqq k$, then every disjoint completely additive-analytic collection of subsets of $X$ has cardinality $\leqq k$ ( $k$ an infinite cardinal).

Proof. By the theorem, such a collection has a $\sigma$-d.d. Choose a point from each nonempty member of this collection, and note that Lemma $6(\S 1.4)$ implies that such a choice set is $\sigma$-discrete. Since $X$ has weight $\leqq k$, every $\sigma$-discrete subset has cardinality $\leqq k$. Hence the original collection can have at most $k$ members.

COROLlARY 7. If $X$ is separable and absolutely analytic, then a disjoint collection of analytic subsets of $X$ is completely additive-analytic in $X$ if and only if it is countable.

Proof. This is just Corollary 6 with $k=\boldsymbol{N}_{0}$ and $0.4(3)$ taken together.

\section{Borel measurable mappings for nonseparable metric spaces.}

3.1 $\sigma$-discrete mappings. A consideration of Theorem 2 (\$2.7) and its applications to the theory of Borel measurable mappings leads us to make the following definitions. A collection $\mathscr{B}$ of subsets of $X$ is said to be a base for the mapping $f: X \rightarrow Y$ if to each open set $V \subset Y$ there corresponds $\mathscr{B}_{V} \subset \mathscr{B}$ with $f^{-1}(V)=\bigcup \mathscr{B}_{V}$. If, in addition, $\mathscr{B}$ is a $\sigma$-discrete collection, then we call $\mathscr{B}$ a $\sigma$-discrete base and we say that the mapping $f: X \rightarrow Y$ is $\sigma$-discrete. It is easily verified that $f: X \rightarrow Y$ is 
$\sigma$-discrete if and only if $f: X \rightarrow f(X)$ is $\sigma$-discrete, and that the restriction of a $\sigma$ discrete mapping is again $\sigma$-discrete. We note that one could also call a mapping " $\sigma$-discretely decomposable" if it had a base which was $\sigma$-d.d. However, this leads to nothing new, since such mappings are also $\sigma$-discrete; and, conversely, $\sigma$-discrete mappings are obviously " $\sigma$-d.d." The former property follows from the fact that a $\sigma$-discrete decomposition of the base yields directly a $\sigma$-discrete base.

3.2 Examples of $\sigma$-discrete mappings. (1) If $Y$ is separable, then every $f: X \rightarrow Y$ is $\sigma$-discrete. For we need only take $\mathscr{B}=\left\{f^{-1}\left(R_{1}\right), f^{-1}\left(R_{2}\right), \ldots\right\}$ where $R_{1}, R_{2}, \ldots$ is a countable open base for $Y$. Conversely, suppose $Y$ has the property that for every $X$ and $f: X \rightarrow Y, f$ is $\sigma$-discrete. Then $Y$ must be separable. Otherwise, we can construct a mapping $f: X \rightarrow Y$ such that $X$ is separable, $f(X)$ is discrete, and $\|f(X)\|=\aleph_{1}$. Accordingly, if $f$ is $\sigma$-discrete, any choice set for the collection of sets $f^{-1}(x)(x \in f(X))$ is an uncountable $\sigma$-discrete set in the separable space $X$; a contradiction.

(2) If $X$ is a $\sigma$-discrete set, then every $f: X \rightarrow Y$ is $\sigma$-discrete. For then we may take $\mathscr{B}=\{\{x\} \mid x \in X\}$. Conversely, a necessary condition for every mapping defined on a fixed metric space $X$ to be $\sigma$-discrete is that $X$ be $\sigma$-discrete. For if $\mathscr{B}$ denotes a $\sigma$-discrete base for a one-to-one correspondence between $X$ and the discrete space of cardinality $\|X\|$, then it follows from the property of a base that each $\{x\}$ must belong to $\mathscr{B}(x \in X)$; that is, $X$ must be $\sigma$-discrete.

(3) Every continuous $f: X \rightarrow Y$, with $X$ or $Y$ metrisable, is $\sigma$-discrete. For we may take $\mathscr{B}=\left\{f^{-1}(V) \mid V \in \mathscr{V}\right\}$ (or $\mathscr{B}=\mathscr{V}$ ) where $\mathscr{V}$ is any $\sigma$-discrete base of open sets for $Y$ (respectively, for $X$ ). That $\mathscr{B}$ is then $\sigma$-discrete (a base for $f$ ) follows easily from the continuity of $f$. (Cf. proof of Lemma 5 (§1.4).)

(4) The composite of two $\sigma$-discrete mappings is $\sigma$-discrete.

For let $f: X \rightarrow Y$ and $g: Y \rightarrow Z$ be $\sigma$-discrete. Let $\mathscr{B}=\bigcup_{n} \mathscr{B}_{n}$ be a $\sigma$-discrete base for $g$ and $\mathscr{C}=\bigcup_{m} \mathscr{C}_{m}$ a $\sigma$-discrete base for $f$, where the collections $\mathscr{B}_{n}$ and $\mathscr{C}_{m}$ are discrete. By the collectionwise normality of $Y$ (see [2]), we can find disjoint open collections $\left\{U_{B} \mid B \in \mathscr{B}_{n}\right\}(n=1,2, \ldots)$ such that $U_{B} \supset B$. Let

$$
\mathscr{C}_{m n}=\left\{C \cap f^{-1}(B) \mid C \in \mathscr{C}_{m}, B \in \mathscr{B}_{n} \text {, and } C \subset f^{-1}\left(U_{B}\right)\right\} \text {. }
$$

Then $\mathscr{C}_{m n}$ is discrete, since each nonvoid $C \subset$ at most one $f^{-1}\left(U_{B}\right)$ for $B$ in $\mathscr{B}_{n}$. We claim that $\bigcup_{m, n} \mathscr{C}_{m n}$ is a base for $g \circ f$. For we have $f^{-1}(B) \subset f^{-1}\left(U_{B}\right)=\bigcup_{m} \cup \mathscr{C}_{m}^{\prime}$ for some $\mathscr{C}_{m}^{\prime} \subset \mathscr{C}_{m}$, and hence $f^{-1}(B)=\bigcup_{m} \cup\left\{C \cap f^{-1}(B) \mid C \in \mathscr{C}_{m}^{\prime}, C \subset f^{-1}\left(U_{B}\right)\right\}$. But the sets $f^{-1}(B)(B \in \mathscr{B})$ are clearly a base for $g \circ f$, and hence so is $\bigcup_{m, n} \mathscr{C}_{m n}$. Since this collection is countable, the proof of (4) is complete.

The fundamental result upon which the significance of this paper is based is the following theorem.

THEOREM 3. If $X$ is absolutely analytic, then a necessary and sufficient condition that a mapping $f: X \rightarrow Y$ be $\sigma$-discrete and have a $\sigma$-discrete base of sets which are of additive class $\alpha$ (resp. analytic) in $X$ is that $f$ be of class $\alpha$ (resp. analytic). The necessity is independent of the hypothesis on $X$. 
Proof. We will prove the analytic case; the proof for the other case is completely analogous.

The condition is necessary. For suppose $f$ is $\sigma$-discrete and has a base $\mathscr{B}=\bigcup \mathscr{B}_{n}$ $(n=1,2, \ldots)$ where $\mathscr{B}_{n}$ is a discrete collection of analytic sets in $X$. Given open $V \subset Y$, by the definition of a base, there exists, for each $n, \mathscr{B}_{n}^{\prime} \subset \mathscr{B}_{n}$ such that $f^{-1}(V)$ $=\bigcup_{n} \cup \mathscr{B}_{n}^{\prime}$. By (3) and (5) of $\S 0.4$, it follows that $f^{-1}(V)$ is analytic in $X$, proving that $f$ is an analytic mapping.

The condition is sufficient. For suppose $f: X \rightarrow Y$ is analytic. Let $\mathscr{V}=\bigcup \mathscr{V}_{n}$ $(n=1,2, \ldots)$ be an open base for $Y$, where each $\mathscr{V}_{n}$ is a discrete collection. We fix $n$ throughout the rest of the argument. It suffices to produce a $\sigma$-discrete collection $\mathscr{B}_{n}$ of analytic subsets of $X$ such that $f^{-1}(V)$ is the union of some subcollection of $\mathscr{B}_{n}$ for each $V$ belonging to $\mathscr{V}_{n}$.

Since $X$ is absolutely analytic,

$$
f^{-1}\left(\mathscr{V}_{n}\right)=\left\{f^{-1}(V) \mid V \in \mathscr{V}_{n}\right\}
$$

is a disjoint completely additive-analytic collection of sets in $\tilde{X}$ (a completion of $X)$. Thus we may apply Theorem $2(\$ 2.7)$ to this collection. By Lemma $4(\$ 1.4)$, there exist open discrete collections $\left\{U_{V m} \mid V \in \mathscr{V}_{n}\right\}(m=1,2, \ldots)$ such that $f^{-1}(V) \subset \bigcup_{m} U_{V m}$ for each $V$ in $\mathscr{V}_{n}$. But then,

$$
\mathscr{B}_{n}=\bigcup_{m}\left\{f^{-1}(V) \cap U_{V m} \mid V \in \mathscr{V}_{n}\right\}
$$

is a $\sigma$-discrete collection of analytic subsets of $X$, and $f^{-1}(V)=\bigcup_{m} f^{-1}(V) \cap U_{V m}$ for each $V$ in $\mathscr{V}_{n}$ as required. It follows that $\mathscr{B}=\bigcup_{n} \mathscr{B}_{n}$ is a $\sigma$-discrete base for $f$ having the desired properties. The theorem is thereby proved.

3.3. We now proceed to show that a substantial part of the basic theory of Borel measurable mappings can be extended, from the "classical" separable metric case, to general metric spaces for the class of $\sigma$-discrete mappings. The properties of $\sigma$ discrete mappings allow us to extend, to general metric spaces, well-known methods used for separable spaces. The fact that $\sigma$-discrete bases (for mappings) can replace countable bases in these arguments is largely a consequence of the properties of "locally Borel" sets (cf. 0.4(4) and 0.4(5)). Of course, the importance of such results is based upon Theorem 3 of the preceding section; namely that a Borel measurable mapping is $\sigma$-discrete whenever it is defined on an absolutely analytic set.

We begin with the following basic lemma.

3.4 LEMMA 10. If $f: X \rightarrow Y$ is $\sigma$-discrete and of class $\alpha>0$ (resp. is analytic, or has the Baire property), then $f$ has a $\sigma$-discrete base of sets which are of ambiguous class $\alpha$ (resp. are analytic, or have the Baire property) in $X$. Moreover, we can take each discrete collection to be metrically discrete.

Proof. We will give the proof for mappings of class $\alpha$; the proofs for the other properties are similar but easier. Let $\mathscr{B}=\bigcup_{m} \mathscr{B}_{m}$ be a $\sigma$-discrete base for $f$, and let 
$\mathscr{V}=\bigcup_{n} \mathscr{V}_{n}$ be a $\sigma$-discrete open base for $Y$, where $\mathscr{B}_{m}$ and $\mathscr{V}_{n}$ are discrete systems for each $m$ and $n(=1,2, \ldots)$. For each $V$ in $\mathscr{V}$ write $V=\bigcup F_{V p}(p=1,2, \ldots)$ where $F_{V p}$ is closed in $Y$. By hypothesis, $f^{-1}\left(F_{V p}\right)$ is of multiplicative class $\alpha$ and $f^{-1}(V)$ is of additive class $\alpha$, and we have $f^{-1}\left(F_{V p}\right) \subset f^{-1}(V)$. Consequently, there exist [6, p. 350] sets $E_{V p}$ of ambiguous class $\alpha$ such that $f^{-1}\left(F_{V p}\right) \subset E_{V p} \subset f^{-1}(V)$. Accordingly, for each triple $(m, n, p)$ of natural numbers, we define

$$
\mathscr{B}_{m n p}=\left\{\bar{B} \cap E_{V p} \mid B \in \mathscr{B}_{m}, V \in \mathscr{V}_{n} \text {, and } B \subset f^{-1}(V)\right\} \text {. }
$$

Since the sets $\bar{B}$ for $B$ in $\mathscr{B}_{m}$ form a discrete collection, which fact follows easily from the discreteness of $\mathscr{B}_{m}$, and each nonempty $B$ in $\mathscr{B}_{m}$ is contained in at most one $f^{-1}(V)$ for $V$ in $\mathscr{V}_{n}$, it follows that $\mathscr{B}_{m n p}$ is discrete in $X$. Thus it suffices to show that $\bigcup_{(m, n, p)} \mathscr{B}_{m n p}$ is a base for $f$.

We will show that

$$
f^{-1}(V)=\bigcup\left\{\bar{B} \cap E_{V p} \mid B \in \mathscr{B}, B \subset f^{-1}(V), p=1,2, \ldots\right\} .
$$

The inclusion $\supset$ is evident from the fact that $E_{V p} \subset f^{-1}(V)$ for all $p$. On the other hand, $f^{-1}(V)=\bigcup\left\{B \mid B \in \mathscr{B}, B \subset f^{-1}(V)\right\}$, since $\mathscr{B}$ is a base for $f$, and $f^{-1}(V)$ $=\bigcup_{p=1}^{\infty} f^{-1}\left(F_{V p}\right) \subset \bigcup_{p=1}^{\infty} E_{V p}$ by construction. Hence the inclusion $\subset$ follows and equality must hold. Since $\mathscr{V}$ is an open base for $Y$, it follows that $\bigcup_{(m, n, p)} \mathscr{B}_{m n p}$ is a base for $f$. To obtain metrically discrete collections one uses the same technique used in the proof of Lemma 2 (\$1.2).

3.5 Complex and product mappings. Given mappings $f: X \rightarrow Y, g: X \rightarrow Z$, we can consider the "complex mappings" [6, p. 382] $h: X \rightarrow Y \times Z$ defined by the equation $h(x)=(f(x), g(x))$.

THEOREM 4. Suppose $f$ is $\sigma$-discrete. Then a necessary and sufficient condition for the mapping $h$ to be of class $\alpha$ is that the "coordinate" mappings $f$ and $g$ be of class $\alpha$.

In particular, this equivalence holds if $f$ is continuous or $X$ is absolutely analytic.

Proof. That the condition is necessary is well known [6, p. 382] and easy to prove; one has only to note that the mappings $f$ and $g$ can be expressed as the composite of $h$ and a continuous projection.

The condition is sufficient. Let $\mathscr{B}=\bigcup \mathscr{B}_{n}(n=1,2, \ldots)$ be a $\sigma$-discrete base for $f$. By the preceding lemma, or by the continuity of $f$ in the case $\alpha=0$, we may suppose that each member of this base is of additive class $\alpha$ in $X$. Let $U=\bigcup_{s}\left\{V_{s} \times W_{s}\right\}$ be an arbitrary open set in $Y \times Z$, where $V_{s}$ and $W_{s}$ are open in $Y$ and $Z$ respectively. We need to show that $h^{-1}(U)$ is of additive class $\alpha$ in $X$. Now we have that $h^{-1}(U)$ $=\bigcup_{s} h^{-1}\left(V_{s} \times W_{s}\right)=\bigcup_{s} f^{-1}\left(V_{s}\right) \cap g^{-1}\left(W_{s}\right)$. Also, letting $\mathscr{B}_{n}=\left\{B_{n t}\right\}_{t \in T_{n}}$, we have $f^{-1}\left(V_{s}\right)=\bigcup_{n} \bigcup_{t \in T_{n}^{\prime}(s)} B_{n t}$ for suitable $T_{n}^{\prime}(s) \subset T_{n}$. It follows that

$$
\begin{aligned}
h^{-1}(U) & =\bigcup_{n} \bigcup_{s} \bigcup_{t \in T_{n}^{\prime}(s)} B_{n t} \cap g^{-1}\left(W_{s}\right) \\
& =\bigcup_{n} \bigcup_{t \in T_{n}} B_{n t} \cap\left(\bigcup_{s \text { with } t \in T_{n}^{\prime}(s)} g^{-1}\left(W_{s}\right)\right) \\
& =\bigcup_{n} \bigcup_{t \in T_{n}} B_{n t} \cap g^{-1}\left(\bigcup_{s w \text { wht teT }} W_{n}^{\prime}(s)\right.
\end{aligned}
$$


Since the sets $B_{n t} \cap g^{-1}\left(\bigcup_{s \text { with } t \in T_{n}^{\prime}(s)} W_{s}\right)$ with $t \in T_{n}$, for fixed $n$, form a discrete collection of sets of additive class $\alpha$ in $X$, their union is also of this class by 0.4(5). Since a countable union of sets of additive class $\alpha$ is again of this class by 0.4(3), it follows that $h^{-1}(U)$ is of the desired class.

The particular cases follow from (3) and Theorem 3 of $\$ 3.2$.

Corollary (Montgomery [10]). If $f: X \rightarrow Y$ is of class $\alpha$, then Graph $(f)$ $=\{(x, y) \mid y=f(x)\}$ is of multiplicative class $\alpha$ in $X \times Y$.

Proof. Let $h: X \times Y \rightarrow Y \times Y$ denote the complex mapping with coordinates $f \circ \pi_{1}$ and $\pi_{2}$, where $\pi_{1}$ and $\pi_{2}$ denote the first and second projection, respectively, of the product space $X \times Y$. Since $\pi_{2}$ is continuous and $f \circ \pi_{1}$ is of class $\alpha$, it follows from the preceding theorem that $h$ is of class $\alpha$. Letting $d$ denote the metric on $Y$, it follows that the composition $d \circ h$ is of class $\alpha$, since $d$ is a continuous mapping on $Y \times Y$. Consequently,

$$
\begin{aligned}
(d \circ h)^{-1}(0) & =\{(x, y) \mid d(f(x), y)=0\} \\
& =\{(x, y) \mid f(x)=y\}
\end{aligned}
$$

is of multiplicative class $\alpha$ in $X \times Y$, which was to be shown.

THEOREM 5. A necessary and sufficient condition for a mapping $h$ from a metric space $X$ to a countable product space $\prod Y_{i}(i=1,2, \ldots)$ to be $\sigma$-discrete and of class $\alpha$ is that for each $i$ the mapping $\pi_{i} \circ h$ be $\sigma$-discrete and of class $\alpha$, where $\pi_{i}$ denotes the ith projection of $\prod Y_{i}$.

In particular, if $X$ is absolutely analytic and each $\pi_{i} \circ h$ is of class $\alpha$, then $h$ is of class $\alpha$.

Proof. The condition is necessary. For this follows from the fact that $\pi_{i} \circ h$ is $\sigma$-discrete (by 3.2(4)) and of class $\alpha$ (by $0.5(9)$ ).

The condition is sufficient. The case $\alpha=0$ reduces to the case $\alpha=1$ and a standard fact about continuity and product spaces. We suppose that $\alpha>0$. By Theorem 3 (\$3.2), it suffices to produce a $\sigma$-discrete base $\mathscr{B}$ for $h$ whose members are of additive class $\alpha$ in $X$. By hypothesis and Lemma 10 (\$3.4), each of the mappings $\pi_{i} \circ h$ has a $\sigma$-discrete base $\mathscr{B}_{i}=\left\{B_{n t}^{i} \mid t \in T_{n}^{i}, n=1,2, \ldots\right\}$ of sets of additive class $\alpha$ in $X$, where the sets $B_{n t}^{i}$ for fixed $n$ form a discrete collection. We consider for each $i=1,2, \ldots$ and for each $i$-tuple $\left(n_{1}, \ldots, n_{i}\right)$ of natural numbers all sets of the form

$$
B_{n_{1} t_{1}}^{1} \cap \cdots \cap B_{n_{t} t_{t}}^{i}
$$

for $\left(t_{1}, \ldots, t_{i}\right) \in T_{n_{1}}^{1} \times \cdots \times T_{n_{i}}^{i}$, and denote the totality of such sets by $\mathscr{B}_{n_{1} \ldots n_{i}}$. Each set of the form (') is of additive class $\alpha$ in $X\left[6\right.$, p. 346]. We take $\mathscr{B}=\bigcup \mathscr{B}_{n_{1} \ldots n_{i}}$ (where the union is over all such $i$-tuples). Now each $\mathscr{B}_{n_{1} \ldots n_{i}}$ is discrete in $X$. For given any $x$ in $X$ there exist neighborhoods $U_{n_{r}}^{r}(r=1, \ldots, i)$ of $x$ such that $U_{n_{r}}^{r}$ meets at most one member of the collection $\mathscr{B}_{n_{r}}^{r}$. Consequently, $U_{n_{1}}^{1} \cap \cdots \cap U_{n_{1}}^{i}$ is a neighborhood of $x$ which meets at most one of the sets in $\mathscr{B}_{n_{1} \ldots n_{l}}$. Since the set 
of all finite sequences of natural numbers is countable, it remains only to show that $\mathscr{B}$ is a base for $h$.

Now $\prod Y_{i}$ has a base of open sets of the form $\pi_{1}^{-1}\left(V_{1}\right) \cap \cdots \cap \pi_{i}^{-1}\left(V_{i}\right)$ where $V_{r}$ is open in $Y_{r}, r=1,2, \ldots, i$. Hence it suffices to show that the inverse image of these sets under $h$, which take the form

$$
\left(\pi_{1} \circ h\right)^{-1}\left(V_{1}\right) \cap \cdots \cap\left(\pi_{i} \circ h\right)^{-1}\left(V_{i}\right),
$$

are expressible as the union of some subcollection of $\mathscr{B}$. By the definition of $\mathscr{B}_{r}$, we have, for $r=1, \ldots, i$,

$$
\left(\pi_{r} \circ h\right)^{-1}\left(V_{r}\right)=\bigcup\left\{B_{n_{r} t_{r}}^{r} \mid t_{r} \in S_{n_{r}}^{r}, n_{r}=1,2, \ldots\right\}
$$

for suitable $S_{n_{r}}^{r} \subset T_{n_{r}}^{r}$. But then (") is equivalent to

$$
\bigcup_{n_{1} \ldots n_{t}} \bigcup_{t_{1} \ldots t_{i}} B_{n_{1} t_{1}}^{1} \cap \cdots \cap B_{n_{i} t_{t}}^{i}
$$

where $\left(n_{1}, \ldots, n_{i}\right)$ ranges over all $i$-tuples of natural numbers and $\left(t_{1}, \ldots, t_{i}\right)$ $\in S_{n_{1}}^{1} \times \cdots \times S_{n_{i}}^{i}$. This proves that $\mathscr{B}$ is a base for $h$ and completes the proof of the theorem.

Definition. If $g_{i}: X_{i} \rightarrow Y_{i}(i=1,2, \ldots)$ and $x=\left(x_{1}, x_{2}, \ldots\right)$ is a point of the product $\Pi X_{i}$, then we define the "product" mapping $p: \prod X_{i} \rightarrow \prod Y_{i}$ by the condition $p(x)=\left(g_{1}\left(x_{1}\right), g_{2}\left(x_{2}\right), \ldots\right)$.

THEOREM 6. If each $g_{i}$ is $\sigma$-discrete and of class $\alpha$, then so is $p$. In particular, if each $X_{i}$ is absolutely analytic and each $g_{i}$ is of class $\alpha$, then $p$ is of class $\alpha$.

Proof. Since $\pi_{i} \circ p=g_{i} \circ \pi_{i}^{\prime}$, where $\pi_{i}^{\prime}$ and $\pi_{i}$ denote the $i$ th projection of $\prod X_{i}$ and $\prod Y_{i}$ respectively, and $g_{i} \circ \pi_{i}^{\prime}$ is $\sigma$-discrete by 3.2(4) and of class $\alpha$ by $0.5(9)$, Theorem 6 follows from Theorem 5 with $p=h$.

THEOREM 7. If $f_{i}: X_{i} \rightarrow Y, i=1,2, \ldots$, is $\sigma$-discrete and of class $\alpha$, then the set $I$ of all points $x \in X_{1} \times X_{2} \times \cdots$ such that $f_{1}\left(x_{1}\right)=f_{2}\left(x_{2}\right)=\cdots$ is of multiplicative class $\alpha$ in $X_{1} \times X_{2} \times \cdots$. Further, the mapping $f$ defined by the condition $f(x)=f_{1}\left(x_{1}\right)$, for $x$ in $I$, is $\sigma$-discrete and of class $\alpha$ from I onto $f(I)=f_{1}\left(X_{1}\right) \cap f_{2}\left(X_{2}\right) \cap \cdots$.

Moreover, if the mappings $f_{i}$ are generalized homeomorphisms of class $(\alpha, \beta)$ and either $f(I)$ is absolutely analytic or each $f_{i}^{-1}$ is $\sigma$-discrete, then $f$ is a generalized homeomorphism of class $(\alpha, \beta)$ and has a $\sigma$-discrete inverse (in which case we say that $f$ is bi- $\sigma$-discrete).

Proof. By the preceding theorem, the product mapping $p: X_{1} \times X_{2} \times \cdots \rightarrow Y \times Y$ $\times \cdots$ is of class $\alpha$. Since the diagonal $D=\left\{y \mid y_{1}=y_{2}=\cdots\right\}$ is a closed subset of $Y \times Y \times \cdots[6$, p. 153], it follows that

$$
p^{-1}(D)=\{x \mid p(x) \in D\}=I
$$

is of multiplicative class $\alpha$ in $X_{1} \times X_{2} \times \cdots$. Furthermore, since $p$ is $\sigma$-discrete (Theorem 6) and the first projection $\pi_{1}$ for $Y \times Y \times \cdots$ is continuous, $\pi_{1} \circ p \mid I=f$ is $\sigma$-discrete and of class $\alpha$. 
We proceed to the proof of the part of the theorem concerning generalized homeomorphisms. It is easy to see [6, p. 153, Theorem 4] that if the mappings $f_{i}$ are one-to-one, the same is true of $f$. Now suppose each $f_{i}$ is of class $(\alpha, \beta)$, and each $f_{i}^{-1}$ is $\sigma$-discrete (or $f(I)$ is absolutely analytic). Then the complex mapping $h: f(I) \rightarrow X_{1} \times X_{2} \times \cdots$ with coordinates $f_{1}^{-1} \mid f(I)$ is $\sigma$-discrete and of class $\beta$ by Theorem 5. But, if $x \in I$, then

$$
\begin{aligned}
h(f(x)) & =\left(f_{1}^{-1}\left(f_{1}\left(x_{1}\right)\right), \ldots, f_{n}^{-1}\left(f_{1}\left(x_{1}\right)\right), \ldots\right) \\
& =\left(f_{1}^{-1}\left(f_{1}\left(x_{1}\right)\right), \ldots, f_{n}^{-1}\left(f_{n}\left(x_{n}\right)\right), \ldots\right) \\
& =\left(x_{1}, \ldots, x_{n}, \ldots\right)=x .
\end{aligned}
$$

Hence $h=f^{-1}$, proving that $f$ is a generalized homeomorphism of class $(\alpha, \beta)$ and is bi- $\sigma$-discrete.

REMARK. The reader can find a complete account of the standard treatment of the concepts of $\S 3.5$ in Kuratowski [6, pp. 382-384].

3.6 The basic theorem for nonseparable Borel sets. Throughout this section, the letter $k$ will denote an infinite cardinal.

Theorem 7 of $\$ 3.5$ can be used to obtain a sharpening of the "basic theorem" for the structure theory of nonseparable Borel sets due to A. H. Stone:

TheOREM (Stone [13, TheORem 4]). If $Y$ is an absolute Borel set of class $\alpha \geqq 1$, and of weight $\leqq k$, there exists a generalized homeomorphism $f$, of class $\left(0, \omega_{0}^{\alpha}\right)$, of some closed subset $A$ of $B(k)$ onto $Y$.

We need the following lemmas which were proven in [13, Lemma 3.3 and Theorem 2].

LEMMA (STONE). If $X$ is a complete metric space of weight $\leqq k$, there exists a generalized homeomorphism $f$, of class $(0,1)$, of a $G_{\delta}$ subset $A$ of $B(k)$ onto $X$.

LEMMA (STONE). Every $G_{\delta}$ subset of $B(k)$ is homeomorphic to a closed subset of $B(k)$.

THEOREM 8. Let $Y$ be a complete metric space of weight $\leqq k$. For every set $X \subset Y$ of multiplicative (resp. ambiguous) class $\alpha \geqq 2$ in $Y$ there exists a generalized homeomorphism $f$, of class $(0, \alpha)$, of a closed subset $A$ of $B(k)$ onto $Y$ such that $f^{-1}(X)$ is a $G_{\delta}\left(\right.$ resp. $F_{\sigma}$ and $G_{\delta}$ ) in $A$. Further, if $\alpha=\beta+1$, then $f$ can be taken to be of class $(0, \beta) .(C f .[6, p .448$, Theorem 1].)

Proof. We consider first the proof for ambiguous classes. We assume then that the theorem is true for all sets of ambiguous class $<\alpha(\alpha \geqq 2)$, and proceed to deduce it for any set $X$ of ambiguous class $\alpha$.

By 0.4(7), $X$ has the form

$$
X=\bigcup_{n=1}^{\infty}\left(X_{n} \cap X_{n+1} \cap \cdots\right)=\bigcap_{n=1}^{\infty}\left(X_{n} \cup X_{n+1} \cup \cdots\right)
$$


where $X_{n}$ is of ambiguous class $\alpha_{n}$ and $0<\alpha_{n}<\alpha$. Then, by the inductive hypothesis (if $\alpha_{n} \geqq 2$ ) or by the preceding two lemmas (if $\alpha_{n}=1$ ), there exist for each $n$ a closed subset $F_{n}$ of $B(k)$ and a generalized homeomorphism $g_{n}$, of class $\left(0, \alpha_{n}\right)$, of $F_{n}$ onto $X_{n}$ (for $\alpha_{n}=1, X_{n}$ is a $G_{\delta}$ in $Y$ and hence completely metrisable [6, p. 408], so the preceding lemmas can be applied). Similarly, since $Y \sim X_{n}$ is of ambiguous class $\alpha_{n}$, there exists a closed subset $K_{n}$ of $B(k)$ and a generalized homeomorphism $h_{n}$, of class $\left(0, \alpha_{n}\right)$, of $K_{n}$ onto $Y \sim X_{n}$.

By 0.6(10), we may identify the discrete union of $F_{n}$ and $K_{n}$ with a closed subset $A_{n}$ of a copy $B_{n}$ of $B(k)$, and define a mapping $f_{n}: A_{n} \rightarrow Y$ by requiring that $f_{n} \mid F_{n}=g_{n}$ and $f_{n} \mid K_{n}=h_{n}$. Evidently, $f_{n}$ is a one-to-one continuous mapping and satisfies the conditions $f_{n}\left(F_{n}\right)=X_{n}$ and $f_{n}\left(K_{n}\right)=Y \sim X_{n}$. Moreover, the mapping $f_{n}^{-1}$ is of class $\alpha_{n}$, since the restrictions $f_{n}^{-1} \mid X_{n}$ and $f_{n}^{-1} \mid Y \sim X_{n}$ are of class $\alpha_{n}$ and $X_{n}$ is ambiguous of class $\alpha_{n}$ in $Y$ [6, p. 377].

Let $B=B_{1} \times B_{2} \times \cdots$ and let $A$ be the set of all points $x$ in $B$ such that $f_{1}\left(x_{1}\right)$ $=f_{2}\left(x_{2}\right)=\cdots$. We define $f$ by the rule $f(x)=f_{1}\left(x_{1}\right)$ for $x \in A$. Since, by Theorem 3 (§3.2), $f_{n}$ and $f_{n}^{-1}$ are $\sigma$-discrete for each $n$, and $f_{n}$ is a generalized homeomorphism of class $\left(0, \alpha_{n}\right)\left(\alpha_{n}<\alpha\right)$, it follows from Theorem $7(\S 3.5)$ that $A$ is a closed subset of $A_{1} \times A_{2} \times \cdots$ (hence of $B$ ) and $f$ is a generalized homeomorphism of class $(0, \alpha)$, if $\alpha$ is a limit ordinal, and of class $(0, \beta)$, if $\alpha=\beta+1$, of $A$ onto

$$
f_{1}\left(A_{1}\right) \cap f_{2}\left(A_{2}\right) \cap \cdots=Y \text {. }
$$

Since $B$ is homeomorphic to $B(k)\left[13\right.$, p. 5], it remains only to show that $f^{-1}(X)$ is an $F_{\sigma}$ and $G_{\delta}$ (we remark that the proof now follows verbatim the one given in the separable case [6, p. 449], although we include it here for the sake of completeness). We have

$$
\begin{aligned}
f^{-1}(X) & =\bigcup_{n=1}^{\infty}\left[f^{-1}\left(X_{n}\right) \cap f^{-1}\left(X_{n+1}\right) \cap \cdots\right] \\
& =\bigcap_{n=1}^{\infty}\left[f^{-1}\left(X_{n}\right) \cup f^{-1}\left(X_{n+1}\right) \cup \cdots\right] ;
\end{aligned}
$$

and since $f$ is continuous, we need show only that $f^{-1}\left(X_{n}\right)$ is closed and open in $A$. But, for $x \in A$, we have

$$
f(x) \in X_{n} \equiv f_{n}\left(x_{n}\right) \in X_{n} \equiv x_{n} \in F_{n}
$$

since $F_{n}$ is both open and closed in $A_{n}$, it follows that

$$
f^{-1}\left(X_{n}\right)=A \cap\left(A_{1} \times \cdots \times A_{n-1} \times F_{n} \times A_{n+1} \times \cdots\right)
$$

is open and closed in $A$.

We proceed to prove the theorem in the case of multiplicative classes. If $X$ is of multiplicative class $\alpha(>1)$, then we have $X=X_{1} \cap X_{2} \cap \cdots$, where each $X_{n}$ is ambiguous of class $\alpha$. By what we have just proved, there exists for each $n$ a closed subset $A_{n}$ of a copy of $B(k)$, denote this copy by $B_{n}$, and a generalized homeomor- 
phism $f_{n}$, of class $(0, \alpha)$, of $A_{n}$ onto $Y$ such that $f_{n}^{-1}\left(X_{n}\right)$ is an $F_{\sigma}$ and $G_{\delta}$ in $A_{n}$. Now let $A$ and $f$ be defined as above. Then

$$
\begin{aligned}
f(A) & =f_{1}\left(A_{1}\right) \cap f_{2}\left(A_{2}\right) \cap \cdots=Y, \\
f^{-1}(X) & =f^{-1}\left(X_{1}\right) \cap f^{-1}\left(X_{2}\right) \cap \cdots,
\end{aligned}
$$

and

$$
f^{-1}\left(X_{n}\right)=A \cap\left(B_{1} \times \cdots \times B_{n-1} \times f_{n}^{-1}\left(X_{n}\right) \times B_{n+1} \times \cdots\right),
$$

which proves that $f^{-1}\left(X_{n}\right)$, and hence $f^{-1}(X)$, is a $G_{\delta}$ in $A$. Finally, if $\alpha=\beta+1$, then, as the above argument shows, we may take $f$ to be of class $(0, \beta)$.

3.7 Continuity apart from sets of the first category. Here we are concerned with the extension to general metric spaces of theorems, well known for the separable case, regarding the continuity properties of Borel measurable mappings or, more generally, mappings which have the Baire property. A complete account of the basic results of the standard theory can be found in [6, pp. 394-403] and [4, pp. 286-291].

THEOREM 9. Let $f: X \rightarrow Y$ be o-discrete. Then a necessary and sufficient condition for $f$ to have the Baire property is that there exists a set $P$ of the first category (in $X$ ) such that the restriction of $f$ to $X \sim P$ is continuous. The sufficiency is independent of the hypothesis on $f[6, p .400]$.

Proof. We need to define a set $P$ of the first category such that the mapping $g=f \mid X \sim P$ is continuous; i.e., $g^{-1}(V)$ is open relative to $X \sim P$ whenever $V$ is open in $Y$. Let $\mathscr{B}=\bigcup_{n} \mathscr{B}_{n}$ be a $\sigma$-discrete base for $f$ such that each $\mathscr{B}_{n}$ is a discrete collection of sets having the Baire property in $X(n=1,2, \ldots)$; such a base exists by Lemma 10 (\$3.4). Accordingly, each $B$ in $\mathscr{B}$ has the form $B=\left(W_{B} \sim P_{B}\right) \cup R_{B}$, where $W_{B}$ is open in $X$ and $P_{B}$ and $R_{B}$ are of the first category (see [6, p. 87]); and we may evidently assume that $P_{B} \subset B$. As the union of a discrete collection of sets of the first category, it follows from a theorem of Banach [12, p. 182] that each of the sets $P_{n}=\bigcup\left\{P_{B} \mid B\right.$ in $\left.\mathscr{B}_{n}\right\}$ and $R_{n}=\bigcup\left\{R_{B} \mid B\right.$ in $\left.\mathscr{B}_{n}\right\}$ is of the first category in $X$, $n=1,2, \ldots$ Let $P=\left(P_{1} \cup R_{1}\right) \cup\left(P_{2} \cup R_{2}\right) \cup \ldots$. Now let $V$ be open in $Y$, and note that $g^{-1}(V)=f^{-1}(V) \sim P$. Hence there exist subcollections $\mathscr{B}_{n}^{\prime} \subset \mathscr{B}_{n}$ such that

$$
g^{-1}(V)=\bigcup_{n}\left[\bigcup \mathscr{B}_{n}^{\prime}\right] \sim P=\bigcup_{n} \bigcup_{B \in \mathscr{P}_{n}^{\prime}}\left[\left(W_{B} \sim P_{B} \cup R_{B}\right) \sim P\right]
$$

Since $P_{B} \cup R_{B} \subset P$, it follows that

$$
\left[\left(W_{B} \sim P_{B}\right) \cup R_{B}\right] \sim P=W_{B} \sim P .
$$

Hence $g^{-1}(V)=\bigcup_{n} \bigcup_{B \in \mathscr{D}_{n}^{\prime}} W_{B} \sim P$ is open relative to $X \sim P$, which proves that $f \mid X \sim P$ is continuous.

REMARK. Whether or not Theorem 9 continues to hold without the $\sigma$-discrete condition remains unanswered (cf. [7, p. 545]). 
COROllaRY 8. If $X$ is absolutely analytic and $f: X \rightarrow Y$ is (in particular) Borel measurable, then there exists a set $P$ of the first category in $X$ such that $f \mid X \sim P$ is continuous.

For Borel measurable mappings of class 1, Theorem 9 takes a much stronger form; and we have the following generalization to nonseparable spaces of a famous theorem of Baire (cf. [6, p. 394, Theorem 1] and [4, p. 289]).

THEOREM 10. The set $D$ of points of discontinuity of a $\sigma$-discrete Borel measurable mapping of class 1 is of the first category.

Proof. Let $f: X \rightarrow Y$ be $\sigma$-discrete and of class 1 . Then $f$ has a $\sigma$-discrete base $\mathscr{B}=\bigcup_{n} \mathscr{B}_{n}$, where $\mathscr{B}_{n}$ is a discrete collection of $F_{\sigma}$-sets in $X$ (Lemma $10, \S 3.4$ ). Now recall that the set $D$ of points of discontinuity of $f$ satisfies the formula $D=\bigcup_{V}\left(f^{-1}(V) \sim\right.$ Int $\left.\left[f^{-1}(V)\right]\right)$, where $V$ runs over the family of open sets of $Y$. Thus we have $D \subset \bigcup_{n} \bigcup_{B \in \mathscr{B} n}(B \sim \operatorname{Int}[B])$, as follows easily from the fact that $\mathscr{B}$ is a base for $f$. But, as boundary $F_{\sigma}$-sets, the sets $B \sim \operatorname{Int}[B]$ are of the first category in $X$ [6, p. 81]. Since the sets $B \sim$ Int [ $B]$, for $B$ in $\mathscr{B}_{n}$, form a discrete collection for each $n$, it follows that $\bigcup_{n} \bigcup_{B \in \mathscr{B}_{n}}(B \sim \operatorname{Int}[B])$, and hence $D$ is of the first category.

REMARK. The problem of "analytic representation" (see [6, p. 392]) of Borel measurable mappings for nonseparable metric spaces can also be solved for the class of $\sigma$-discrete mappings. One can show that, for $\alpha>1$, every $\sigma$-discrete mapping $f: X \rightarrow Y$ of class $\alpha$ is the pointwise (uniform) limit of a sequence of $\sigma$-discrete mappings of classes $<\alpha(=\alpha)$, each having a discrete range contained in the range of $f$. Also, if either $\operatorname{dim} X=0$ (in the sense of the Lebesgue covering dimension [11]) or $Y$ is an AR (metric) (i.e., an absolute retract for metric spaces), then the theorem is also true for $\alpha=1$. As a consequence of these results one obtains generalizations of the Banach and Lebesgue-Hausdorff Theorems on analytic representation (see [1], [9], and [6, pp. 392-394]). These results, in turn, can then be applied to obtain extensions to nonseparable spaces of the "classical" theorems on the extension properties of Borel measurable mappings, including a generalized form of the Lavrentiev theorem (cf. [8]). The author hopes to publish the details in a subsequent paper.

ACKNOWLEDGEMENTs. The author would like to acknowledge his indebtedness to Professor A. H. Stone for his expert supervision during the preparation of this paper. Also, I thank the referee for his helpful comments which eliminated several inaccuracies and made the presentation much clearer.

\section{BIBLIOGRAPHY}

1. S. Banach, Uber analytisch darstellbare Operationen in abstrakten Räumen, Fund. Math. 17 (1931), 281-295.

2. R. H. Bing, Metrization of topological spaces, Canad. J. Math. 3 (1951), 175-186. MR 13, 264. 
3. A. G. El'kin, A-sets in complete metric spaces, Dokl. Akad. Nauk SSSR 175 (1967), 517-520= Soviet Math. Dokl. 8 (1967), 874-877. MR 35 \#4881.

4. F. Hausdorff, Mengenlehre, de Gruyter, Berlin, 1937; English transl., Chelsea, New York, 1957. MR 19, 111.

5. J. L. Kelley, General topology, Van Nostrand, Princeton, N. J., 1955. MR 16, 1136.

6. K. Kuratowski, Topologie. Vol. 1, PWN, Warsaw, 1958; English transl., Academic Press New York; PWN, Warsaw, 1966. MR 19, 873; MR 36 \#840.

7. - Quelques problèmes concernant les espaces métriques non-séparables, Fund. Math. 25 (1935), 535.

8. - Sur le prolongement de l'homéomorphie, C. R. Acad. Sci. Paris 197 (1933), 1090.

9. H. Lebesgue, J. Math. 1 (1905), 168.

10. D. Montgomery, Non-separable metric spaces, Fund. Math. 25 (1935), 527-534.

11. K. Morita, Normal families and dimension theory for metric spaces, Math. Ann. 128 (1954), 350-362. MR 16, 501.

12. W. Sierpiński, General topology, 2nd ed., Univ. of Toronto Press, Toronto, 1956.

13. A. H. Stone, Non-separable Borel sets, Rozprawy Mat. 28 (1962). MR 27 \#2435.

14. —-, On o-discreteness and Borel isomorphism, Amer. J. Math. 85 (1963), 655-666. MR 28 \#33.

University of ConNeCticut, Storrs, CONNECTicut 06268 